\title{
Video Article \\ Electroencephalographic, Heart Rate, and Galvanic Skin Response Assessment for an Advertising Perception Study: Application to Antismoking Public Service Announcements
}

\author{
Giulia Cartocci ${ }^{1}$, Myriam Caratù $^{2}$, Enrica Modica ${ }^{3}$, Anton Giulio Maglione ${ }^{1}$, Dario Rossi ${ }^{3}$, Patrizia Cherubino ${ }^{4}$, Fabio Babiloni ${ }^{1}$ \\ ${ }^{1}$ Department of Molecular Medicine, Sapienza University of Rome \\ ${ }^{2}$ Department of Communication and Social Research, Sapienza University of Rome \\ ${ }^{3}$ Department of Anatomical, Histological, Forensic, and Orthopedic Sciences, Sapienza University of Rome \\ ${ }^{4}$ BrainSigns SRL
}

Correspondence to: Giulia Cartocci at giulia.cartocci@brainsigns.com

URL: https://www.jove.com/video/55872

DOI: doi:10.3791/55872

Keywords: Neuroscience, Issue 126, Consumer neuroscience, neuromarketing, alpha, alpha asymmetry, theta, electroencephalography, heart rate, galvanic skin response, approach withdrawal theory, emotional index, emotion

Date Published: 8/28/2017

Citation: Cartocci, G., Caratù, M., Modica, E., Maglione, A.G., Rossi, D., Cherubino, P., Babiloni, F. Electroencephalographic, Heart Rate, and Galvanic Skin Response Assessment for an Advertising Perception Study: Application to Antismoking Public Service Announcements. J. Vis. Exp. (126), e55872, doi:10.3791/55872 (2017).

\section{Abstract}

The evaluation of advertising, products, and packaging is traditionally performed through methods based on self-reports and focus groups, but these approaches often appear poorly accurate in scientific terms. Neuroscience is increasingly applied to the investigation of the neurophysiological bases of the perception of and reaction to commercial stimuli to support traditional marketing methods. In this context, a particular sector or marketing is represented by public service announcements (PSAs). The objective of this protocol is to apply electroencephalography (EEG) and autonomic signal analysis to study responses to selected antismoking PSAs. Two EEG indices were employed: the frontal alpha band EEG asymmetry (the Approach Withdrawal (AW) index) and the frontal theta (effort index). Furthermore, the autonomic Emotional Index (EI) was calculated, as derived from the Galvanic Skin Response (GSR) and Heart Rate (HR) signals. The present protocol describes a series of operational and computational steps required to properly estimate, through the aforementioned indices, the emotional and cerebral reaction of a group of subjects towards a selected number of antismoking PSAs. In particular, a campaign characterized by a symbolic communication style (classified as "awarded" on the basis of the prizes received by specialized committees) obtained the highest approach values, as estimated by the AW index. A spot and an image belonging to the same PSA campaign based on the "fear arousing appeal" and with a narrative/experiential communication style (classified as "effective" on the basis of the economical/health-related improvements promoted) reported the lowest and highest effort values, respectively. This is probably due to the complexity of the storytelling (spot) and to the immediateness of the image (a lady who underwent a tracheotomy). Finally, the same "effective" campaign showed the highest El values, possibly because of the empathy induced by the testimonial and the explicitness of the message.

\section{Video Link}

The video component of this article can be found at https://www.jove.com/video/55872/

\section{Introduction}

As David Ogilvy strongly stated, "People don't think what they feel, don't say what they think and don't do what they say." Therefore, it can be inferred that humans are not rational beings. This assumption is further supported by the evidence that many decisions concerning economic issues are not under direct volitional control but rather depend on automatic, rapid, and efficient cognitive processes ${ }^{1}$. Furthermore, emotional mechanisms can influence these decisional processes, contributing to the undertaking of a particular action ${ }^{2,3,4}$. In this context, on top of the traditional tools used in marketing research ${ }^{5}$, it is ever more important to study techniques that are capable of providing additional information on consumer behavior. This investigation still relies on customers' perceptions/evaluations of products and advertising messages, verbally expressed and self-reported, a method that is susceptible to several biases. In fact, so far, the typical methods used to gain insights into the market performance of a new product or service have been based solely on self-reports and focus groups. However, social psychology and market research studies have recognized that self-reports are not reliable at accurately predicting the customers' preferences ${ }^{6}$. The authors of the abovementioned research have performed a study on pre-market forecasting in the footwear retail industry, determining that self-report-based methods were poorly accurate at foretelling success, while brain data achieved a prediction accuracy of $80 \%$. Consumer neuroscience is a field of study that was born as the answer to this need and consists of the application of typical neuroscience methodologies to the investigation of human behavior relating to market and economic exchanges ${ }^{7}$. The term "neuromarketing" refers to the application of neurophysiological tools-such as eye tracking, skin conductance, heart rate, EEG, and functional magnetic resonance imaging (fMRI)-to market research design according to the needs of different companies 8,9 . The abovementioned techniques are drawing growing interest from various companies: proportionally, the rise of neuromarketing companies over the last decade has been impressive ${ }^{7}$. Neuromarketing, in fact, allows 
for the investigation of several aspects relating to commerce: advertising target definitions in terms of gender ${ }^{10,11}$, culture ${ }^{12,13}$, fragments of the advertisement itself $^{10,14,15}$, age $^{16}$, branding solutions ${ }^{17}$, price architectures ${ }^{18}$, scene targeting and the speaker's gender ${ }^{19}$, customers' purchasing attitudes $^{20}$, and pre-retail testing ${ }^{6}$.

Concerning the EEG, two indices have been employed for assessing the responses returned by the subjects exposed to the advertising stimuli: the AW and the effort indices. The first is rooted in studies by Davidson (1990), which demonstrated that a frontal asymmetry between the activity in the two cerebral hemispheres implies a different motivational tendency towards the proposed stimuli. In particular, several authors ${ }^{21,22}$ have reported the presence of two distinct neural systems, mediating an approach-related motivation and a withdrawal-related motivation, localized in the left and right hemispheres, respectively. According to this evidence, the prefrontal cortex (PFC) plays an important role in the circuitry that implements both the positive and the negative motivation. Specifically, the differential involvement of the left and right PFC, with an increase in the left anterior activation during positive motivation and increased right-sided anterior activation during negative motivation, has been reported by various studies ${ }^{23,24,25}$. Furthermore, various reports ${ }^{13,14}$ highlight the possibility of applying the AW index-defined as the difference in prefrontal activity in the alpha band between the two hemispheres-to the advertising field. In addition to the broad investigation on this topic, research has also been conducted on specific product categories, such as the perfumes ${ }^{10}$, where women reported positive motivation toward the advertising, while men reported negative motivation. The research on the AW index also considered some experimental sample characteristics, such as whether or not the subject was a consumer of the advertised item ${ }^{26}$. For instance, in response to a beer commercial, beer drinkers reported higher levels of PFC activation than those subjects who do not drink beer. Furthermore, another parameter that has been considered is the participants' gender. For example, in response to observing a family car spot, men (unlike women) showed positive motivation for the commercial during the entire duration ${ }^{27}$. A final parameter that has been investigated is the age of experimental subjects. An exhilarating climax with some "not-so-nice" images was more cerebrally appreciated by young people than by adults ${ }^{27}$. To summarize, positive values obtained by the AW index, derived from the alpha frontal asymmetry, indicate higher left activity, suggesting positive motivation toward the stimuli. Vice versa, negative values indicate a higher right activity, suggesting negative motivation in response to the stimuli.

The second EEG index, the cerebral effort (CE), defined as the frontal theta activity in the PFC, has been described in several studies. In particular, these studies show how higher values of CE are linked to higher levels of task difficulty ${ }^{28}$. This has been particularly investigated in neuroaesthetics, in response to auditory literature stimuli ${ }^{29}$; in avionics ${ }^{30}$; in different listening conditions ${ }^{31,32}$; and in human-computer interactions ${ }^{33}$.

As suggested by empirical evidence from various fields of application, emotional involvement is a catalyst for the memorization of stimuli. This phenomenon has also been investigated in neuromarketing, demonstrating that positive or negative emotional processing during the observation of commercial advertisements is an important factor for the formation of stable memory traces ${ }^{34}$. Furthermore, it is worth considering that an "unconscious emotion" occurs when the brain systems that mediate unconscious core "liking" (among the other subcortical regions, the nucleus accumbens and its connections) are not coupled with conscious awareness ${ }^{35}$. At the beginning of the present article, it has been underlined how the perception and reaction to advertising stimuli are frequently unconscious. One of the aims of neuromarketing is to investigate this aspect of the emotional reaction. An autonomic index was created by matching the GSR and the HR because these two autonomic parameters are known to reflect the emotional response to stimuli ${ }^{36}$. The resulting El was conceptualized on the basis of the circumflex model of affect, theorized by Russell and Barrett ${ }^{5}$. In this model, the HR is graphically rendered on the x-axis, while the GSR is on the y-axis, returning information concerning the valence (positive or negative) and arousal (low or high activation) of stimuli ${ }^{37,38}$, respectively. The El has already been applied to the testing of auditory literary stimuli ${ }^{29}$, as well as to TV commercials ${ }^{10,16,19,39}$. Therefore, in the authors' opinion, it has been worthwhile to apply the EI to PSAs, particularly to anti-smoking PSAs.

Every year, there are six million deaths around the world caused by smoking, and the major tool to face this emergency consists of antismoking $\mathrm{PSAs}^{40}$. This kind of PSA has been broadcast since the $1950 \mathrm{~s}$, but the effectiveness on the general public is extremely variable, resulting in a series of effective or ineffective campaigns. Generally, the effectiveness of an antismoking campaign is measured on the basis of the rise in public health achieved after the campaign is aired; changes in attitudes, beliefs, or behavior (i.e., an increase in awareness, a change of negative attitudes, an increase in the number of calls to quit lines, etc.); media impact (i.e., positive discussion and appreciation on social media); and so on. Additionally, standard economic evaluation designs are usually applied, such as cost-effectiveness analysis, cost utility analysis, or costbenefit analysis ${ }^{41}$. Finally, beyond the evidence provided by in-depth, specialized cost-effectiveness evaluations, by comparing the costs for the campaign to the medical costs saved, it is possible to affirm that effective campaigns save some money overall and prevent tens of thousands of premature deaths $\mathrm{s}^{42,43,44}$

The cerebral/emotional reaction to particular PSAs therefore appears worthy of investigation by neurophysiological techniques to obtain measurements of the physiological responses that complement the explicit feedback provided by the more traditional interviews and questionnaires.

The indices adopted in the present study have already been applied to antismoking PSAs in a reduced sample of participants and spots, supporting the potential usefulness of the application of neuromarketing techniques to the investigation of neurophysiological features of effectiveness in PSAs ${ }^{11,45}$.

The aim of this study is to present a series of methodological steps that could lead to the accurate measurement of the cerebral and emotional perception of antismoking PSAs. Such a measure is preliminary to the analysis of the instinctive and physiological underpinnings and of the features of a particular PSA that are effective or ineffective at generating a public reaction.

\section{Protocol}

All experimentation was performed according to the principles outlined in the Declaration of Helsinki of 1975, as revised in 2000, and was approved by the university ethical committee. 


\section{Equipment Mounting and Recording Condition Control}

1. Invite the participant to sit on a comfortable chair in front of a computer screen

2. Clean the skin on the forehead (gently moving the hair, if necessary), the ear lobes, and the fingers with a chlorhexidine antiseptic solution to disinfect the skin and to remove the most superficial lipid layer that has accumulated on the skin ${ }^{46}$.

3. Apply a six-electrode EEG band to the participant's forehead according to the 10-20 international system (AFz, FPz, AF5, AF6, AF7, AF8) ${ }^{47}$.

4. Place the reference and the ground electrode, one per ear lobe.

5. Attach the pulse oximeter to the thumb to record the HR.

6. To collect the GSR, place two electrodes on the second and third fingers of the non-dominant hand. Place the electrodes on the palmar side of the second phalanx (following already published procedures) ${ }^{48}$.

7. Check the impedances of the EEG signal to keep them below $10 \mathrm{k} \Omega$ by using the appropriate impedance-measurement button on the interface in the acquisition software.

1. In the "channels" setup of the acquisition software, set the "sampling rate" for EEG signal acquisition to "250 Hz."

8. Acquire the EEG signal through an EEG amplifier system and a related EEG recording software for EEG data acquisition (see Table of Materials). Start the EEG signal recording by pressing the red recording button on the software interface.

9. Check that the GSR and HR signals are being correctly acquired by verifying the presence of the proper waveform on the software interface.

1. In the "channels" setup of the acquisition software, set the sampling rate for GSR and HR signal acquisition to "128 Hz."

10. Acquire autonomic signals (i.e., GSR and HR) through a multipurpose measuring capability system (see the Table of Materials).

11. Start the GSR and HR signal recording by pressing the "record" button on the corresponding software interface.

12. Ask the participant to look at a white "cross" over a black background, which is displayed on the screen for 1 min. Simultaneously acquire the resting condition activity, signaling its start and end with the relative marker buttons on the EEG acquisition software.

13. Instruct the participant, equipped with the EEG, HR, and GSR recording instrument, to watch the PSA video, during which the signal recordings take place. Furthermore, ask the participant to limit any movement and to stay as relaxed as possible for the duration of the recording.

14. Press the starting marker button on the EEG acquisition software interface at the beginning of the PSA video. Press the ending marker button at the end of the PSA video.

\section{Stimuli}

1. Select the target stimuli, preferably on the basis of predefined key performance indicators (KPIs).

2. When designing the experimental protocol, intersperse the six target antismoking stimuli (i.e., three spots and three images belonging to the three selected PSAs) in a video composed of two blocks.

1. Build the spots block as follows: a spot baseline (i.e., a documentary lasting $1 \mathrm{~min}$ ), a train of 10 antismoking spots (use a maximum of 10 items to resemble a typical TV commercial break), and the spot baseline.

2. Build the images block as follows: an image baseline (i.e., 8 neutral images taken from the International Affective Picture System (IAPS) database ${ }^{49}$ ), a train of 10 antismoking images (for coherence with the spots block), and the image baseline.

3. To avoid the bias of a positional effect in the participants' reactions, start by showing half of the participants the spots block and half of the participants the images block.

To avoid a positional bias at the level of specific items, display the stimuli in a randomized order within each block.

4. Select an appropriate 1-min video baseline (i.e., as emotionally neutral as possible, such as a documentary extract) and place it immediately before and immediately after the spots block in the video protocol.

5. Select a proper image baseline (i.e., as neutral as possible, such as 8 neutral images taken from the IAPS database $)^{49}$ and place it immediately before and immediately after the images block in the video protocol.

6. When designing the images block, set the display duration of each image to $9 \mathrm{~s}$ to ensure the detection of eventual variation in the autonomic components, which present a slower response in comparison to the EEG signal. Between every image set, the display a white cross on a black screen to re-establish the central fixation point.

\section{Signal Processing}

NOTE: The following computational steps could be performed with various publicly available signal processing computational software, such as EEGLAB ${ }^{50}$ or LEDALAB ${ }^{51}$, for the analysis of the GSR signal. While a specifically developed software was used here for some calculations, the steps described below do not depend upon the particular software used. Thus, the following steps will not precisely describe the interaction with the computational software but will rather demonstrate the logical steps needed to achieve the results.

1. EEG signal processing.

1. To detect and remove components due to eye movements, blinks, and muscular artifacts, apply a notch filter ( $50 \mathrm{~Hz})$, a band-pass filter $(2-30 \mathrm{~Hz})$, and the independent component analysis $(\mathrm{ICA})^{52}$ procedure to the EEG traces.

1. Filter the EEG signals with a notch filter $(50 \mathrm{~Hz})$, to reject the main electricity components, and then with a band-pass filter (2-30 $\mathrm{Hz}$ ), to reject frequency components that are not related to the cognitive processes being investigated.

2. Convert the EEG time series in the $\mathrm{ICA}^{52}$.

3. Find and remove the independent components related to artifacts. NOTE: Independent components related to ocular artifacts are clearly distinguishable from cerebral components by their shape and magnitude. Once detected, remove such components related to ocular artifacts from the ICA procedure before recomposing the signal in the time domain. 
4. Recompose the EEG signal in the time domain using the retained independent components.

2. For each subject, estimate the individual alpha frequency (IAF) from the resting condition, to define the frequency bands of interest according to the method suggested in the scientific literature ${ }^{28}$.

NOTE: This step is important, since each definition of the frequency band must be determined for each subject individually.

3. Calculate the global field power (GFP) ${ }^{16}$ for each cortical area and for each subject.

1. Filter the EEG signals in the frequency bands of interest, in particular theta (IAF-6:IAF-2) and alpha (IAF-2:IAF+2), according to the definition suggested in the scientific literature ${ }^{28}$.

2. Calculate the GFP ${ }^{16}$ as the sum of the power of EEG signals from specific electrodes over the cortical area of interest (e.g., the left and right prefrontal cortex. Filter in a certain band and average by the number of the considered electrodes. See the following equation:

$\operatorname{GFP}(\mathrm{t})_{\alpha}=\frac{1}{N} \sum_{1}^{N}\left(x_{i, \alpha}(t)\right)^{2}$.

\section{Approach Withdrawal Index}

NOTE: In several studies, the frontal cortex has been indicated as an area of interest for approach or withdrawal attitude in response to a wide range of stimuli ${ }^{53,54,55,56,57,58}$.

1. Apply the formula defining the AW index as AW = GFPa_right - GFPa_left, where GFPa_right and GFPa_left represent the GFP calculated from the right (AF6 and AF8) and left (AF5 and AF7) electrodes in the alpha band ${ }^{59,60}$

2. Estimate the waveform of the AW cerebral index for each second and then average over the duration of the stimuli.

3. Standardize the AW index according to the baseline EEG activity acquired at the beginning and at the end of the experiment. NOTE: Positive AW values mean an approach motivation toward the stimulus expressed by the subject, while negative AW values indicate a withdrawal tendency.

\section{Effort index}

NOTE: Several studies demonstrate the investigation and application of cerebral effort to various tasks in humans ${ }^{28,33}$.

1. For the evaluation of the cerebral effort, use frontal electrodes in the theta band (AFz, FPz, AF5, AF6, AF7, AF8) ${ }^{28,11,33,45}$.

2. Estimate the GFP ${ }^{16}$ from the frontal electrodes. Standardize the effort index data, as for the AW index, according to the baseline EEG activity acquired at the beginning and at the end of the experiment.

3. For result interpretation, remember that higher levels of effort index imply a higher level of task difficulty ${ }^{31}$.

\section{Emotional Index}

1. To obtain the HR signal, employ the Pan-Tompkins algorithm ${ }^{61}$.

2. Acquire the skin conductance using the constant voltage method $(0.5 \mathrm{~V})^{62}$

3. Use LEDAlab software ${ }^{67}$ to obtain the tonic component of the skin conductance (i.e., skin conductance level (SCL)).

1. Select "Continuous Decomposition Analysis" from the analysis menu.

NOTE: It is recommended to optimize the analysis by clicking the "Optimize" button.

2. Click on "Apply" to accept the analysis and have the decomposition plotted.

4. For the purpose of matching SCL and HR signals, refer to the circumplex model of affect plan ${ }^{63,64}$, where the coordinates of a point in space are defined respectively by the HR (horizontal axis) to describe the valence and by the SCL (vertical axis) to describe the arousal phenomena ${ }^{36}$.

5. To obtain a monodimensional variable, use the emotional state of a subject as described by the $\mathrm{El}^{10}$, defined by the formula: $\mathrm{El}=1-\beta /$ $\pi^{\prime}$. NOTE: Here,

$$
\beta=\left\{\begin{array}{c}
\frac{3}{2} \pi+\pi-\vartheta \text { if } G S R_{z} \geq 0, H R_{z} \leq 0, \\
\frac{\pi}{2}-\vartheta \text { otherwise. }
\end{array}\right\}
$$

$\mathrm{HR}$ and $G S R_{z}$ constitute the Z-score variables of HR and GSR, respectively. $\vartheta$ is in radians, calculated as $\operatorname{arctang}\left(H R_{z} G S R_{z}\right)$. Because the angle will vary between $[-1,1]$, the El will vary between $[-1,1]$.

6. For result interpretation, remember that higher El values imply more positive emotion experienced by the participant, and vice versa.

\section{Representative Results}

22 healthy subjects (mean age $17.64 \pm 0.95$ years, range $=16-19$ years old) were enrolled on a voluntary base. Concerning smoking habits, 7 participants were not smokers, 9 were light smokers ( $\leq 5$ cigarettes per day), and 6 were heavy smokers ( $>5$ cigarettes per day). The smoker participants reported to have smoked their first cigarette at the mean age of $13.38 \pm 3.01$ years old, and none of the participants reported to have quit in the past. All subjects were given detailed information about the study and signed an informed consent. The experiment was performed according to the principles outlined in the Declaration of Helsinki of 1975, as revised in 2000, and was approved by the university ethical committee. 
In the present study, we used the KPIs proposed by Coffman ${ }^{65}$ and Varcoe ${ }^{66}$, and we selected three target antismoking campaigns. Two were included on the basis of being classificatied as "effective" and "ineffective," as evidenced by official data on the promotion of health and on economic improvements in the general population. A third PSA was classified and included on the basis of the appreciation obtained from specialized committees, expressed in term of the amount of prizes received. In specific, the three selected antismoking PSAs in the present study were: i) CDC (image: CDC Terry; spot: CDC Roosevelt) ${ }^{67}$, USA 2012-2015, effective ${ }^{68,44}$, fear-arousing appeal and narrative/experiential communication style; ii) Think don't smoke (image and video) ${ }^{69}$, Philip Morris, USA 1998, ineffective ${ }^{70,71}$, paternalistic communication style; and iii) Fatty Cigarette (image and video) ${ }^{72}$, British Heart Foundation, UK 2003, awarded (Clio Awards 2005 TV and Radio Public Service Bronze, IPA Effectiveness Award 2004 The Winners Golden Watch; IPA Effectiveness Award 2004 The Winners Best Dedication to Effectiveness Winner; and 3 Palmares au Festival International de la Publicite de Cannes 2004), symbolic communication style.

The statistical analysis consisted of an ANOVA, with the category of the PSA as a within-factor with three levels (effective, ineffective, and awarded) and gender (M/F) as a between-factor. Smoking attitude was not included in the analysis because of the low numbers within the three possible groups (i.e., non-smokers, light smokers, and heavy smokers), but the homogeneous distribution of the participants in these three groups allowed for the performance of the analysis in the collapsed sample. It is important to recall that the ANOVA test has sufficient statistical power to deal with the analysis of relatively small numbers of participants, as in this study ${ }^{73}$, provided that the number of factors is lower than 4 , as in this case.

\section{Approach Withdrawal Index:}

For the images, no statistically significant differences between the three campaigns were identified $(F(2,40)=2.649, p=0.083)$, but $A W$ values reported for the "awarded" image were higher than the ones reported for the "ineffective" PSA (Figure 1, left).

For the video stimuli, a statistically significant effect of the spot category (i.e., effective, ineffective, or awarded) was discovered $(F(2,40)=3.171$, $p=0.050)$. The post-hoc analysis highlighted the increased AW values estimated for the "awarded" spot in comparison to the "effective" one ( $p=$ $0.047)$ and an analogously strong tendency $(p=0.060)$ in comparison to the "ineffective" spot (Figure 1, right).

In summary, both the image and the spot belonging to the "awarded" campaign obtained the most positive approach values, as estimated by the AW index.

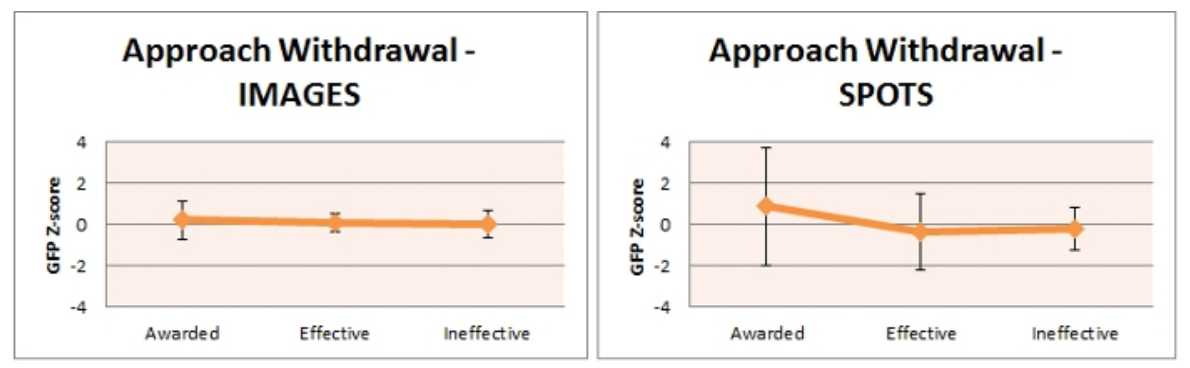

Figure 1: AW index estimation of the selected antismoking PSA campaigns. On the left are results related to the images, and on the right are results related to the spots of the "effective," "ineffective," and "awarded" antismoking PSAs. Error bars represent the standard deviations. Please click here to view a larger version of this figure.

\section{Effort Index:}

The ANOVA test highlighted a statistically significant effect of the category (i.e., effective, ineffective, or awarded) variable for both the images $(F(2,40)=8.589, p=0.001)$ and spot $(F(2,40)=5.441, p=0.008)$ stimuli. The post-hoc analysis revealed that, for the images, the "effective" one was significantly lower than the "ineffective" ( $p=0.009)$ and "awarded" ( $p<0.001)$ ones (Figure 2, left). In addition, the post-hoc analysis performed on the spots showed that the effort values reported for the "effective" video were significantly higher $(p=0.003)$ than the ones estimated for the "awarded" spot (Figure 2, right).

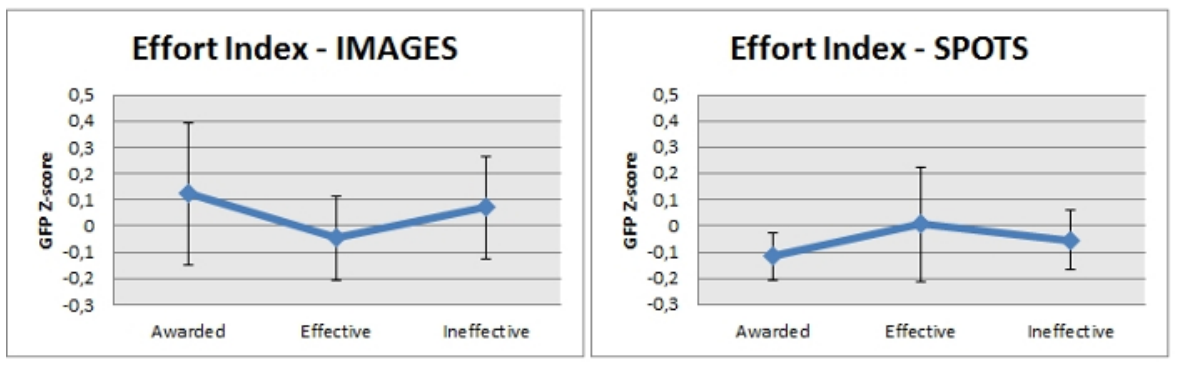

Figure 2: Effort index estimation of the selected antismoking PSA campaigns. On the left are results related to the images, and on the right are results related to the spots of the "effective," "ineffective," and "awarded" antismoking PSAs. Error bars represent the standard deviations. Please click here to view a larger version of this figure. 


\section{Emotional index:}

In general, the EI values reported for the "effective" campaign were higher than those of the "ineffective" and "awarded" ones, both for the antismoking PSA image and spot. Concerning the image stimuli, although there was a lack of significant statistical differences between the evaluated conditions, a strong trend of increasing El values for the "effective" image in comparison to the "ineffective" one can be appreciated (Figure 3, left). For the video stimuli, a statistically significant effect of the spot category factor was found $(F(2,32)=3.978, p=0.029)$. Furthermore, the post-hoc analysis showed a decrease in El values for the "ineffective" spot in comparison to the "effective" one $(p=0.013)$ and a markedly similar tendency $(p=0.060)$ in comparison to the "awarded" spot (Figure 3, right). In general, the El values reported for the "effective" campaign were higher than those of the "ineffective" and the "awarded" ones, both for the image and spot antismoking PSAs.

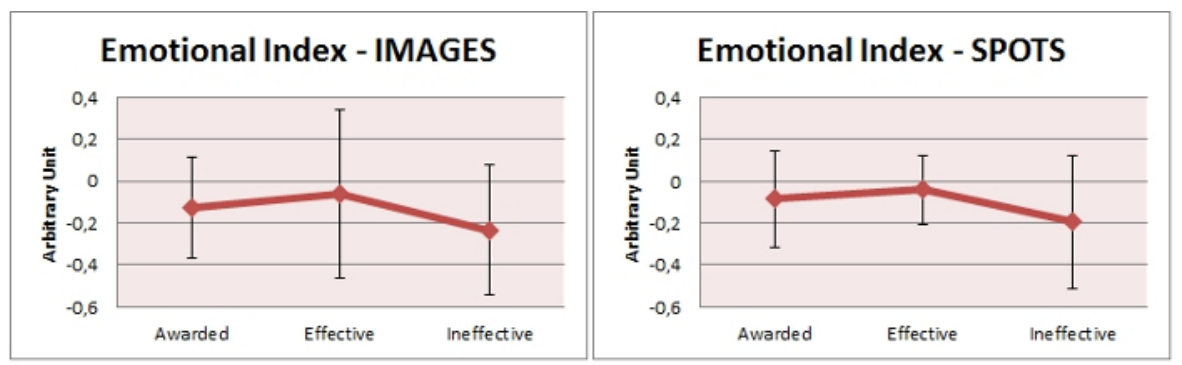

Figure 3: El estimation of the selected antismoking PSA campaigns. On the left results are related to the images, and on the right are results related to the spots of the "effective," "ineffective," and "awarded" antismoking PSAs. Error bars represent the standard deviations. Please click here to view a larger version of this figure.

\section{Discussion}

\section{Modifications of the Protocol and Troubleshooting:}

The protocol described here can be modified for use in a different experimental situation. In particular, the train of stimuli provided here can be changed to a series based only on images or only on videos. This change would allow for tests of only printed or only video advertising, respectively. However, here there are no particular changes in the steps required for signal processing since the occurrence of ocular artifacts will happen in any case and they must be removed from the EEG signals. When only videos are displayed, the baseline could consist only of videos, without the use of images taken from the IAPS database (as described above). A common occurrence necessitating troubleshooting for all EEG recordings is the presence of several muscle artifacts due to subject movement during the recording sessions. In this case, it is important to monitor the quality of the collected EEG traces and to examine the video of the recording session to check for the occurrence of such movement artifacts later. If such artifacts occur, modifications of the procedure to apply the ICA will be necessary to remove such artifacts. Due to the random occurrence of such artifacts along the EEG traces, the ICA procedure can easily produce a component in which such muscle artifacts are present and that can remove them from the successive counter-transformation in the time domain to return clean EEG data.

\section{Limitations of the Technique:}

The cerebral and emotional recording technique described here has several limitations that need to be taken into account when it is applied to experimental procedures different than the one presented. In particular, the ICA procedure is sensitive to the number of the EEG sensors employed. Thus, decreasing the number of sensors to sped up the recordings will impact the number of artifacts that are possible to remove. In fact, the procedure described here includes six sensors and allows for the removal of two main artifact components (e.g., ocular and gross muscle artifacts). The lower the number of EEG sensors used, the lower the number of different kinds of artifacts that can be removed.

Another limitation of the presented technique is related to the total amount of time for the cerebral recordings. In fact, the total duration recommended in similar studies is a maximum of $1 \mathrm{~h}$ for each subject to avoid a possible confounding effect due to tiredness or boredom onset. To this extent, the length of the stimulation must be strictly controlled, and a wide set of stimuli cannot be tested on the same participant, or at least not in a single run. During the $1 \mathrm{~h}$ engagement, the participant must be carefully informed about the study and asked to sign the informed consent, equipped with the experimental instruments, and recorded under resting conditions and during the experimental task sessions. To avoid exceeding the recommended time limit, a possible troubleshooting consideration is to have two experimenters set up the instruments at the same time.

\section{Significance with Respect to Existing Methods:}

The presented protocol for recording and analyzing the cerebral and emotional reaction to PSAs has several advantages in comparison to traditional techniques of advertising evaluation. In particular, one of them is the possibility of using a sample size lower than that necessary to obtain significant results when compilating verbal questionnaires. In fact, while the protocol described here could be fruitfully implemented with a sample size of 20-30 participants, the verbal questionnaire requires at least 100 respondents.

Furthermore, the present technique can investigate physiological responses without relying on the verbal reports of tested participants, providing more instinctive reactions to the stimuli exposure. 


\section{Future Applications:}

The proposed protocol could be applied to a larger sample of subjects to gather further information about the effectiveness of the PSAs. One future application is to identify specific population targets for the a priori prediction of features defining the effectiveness of PSAs to convey appropriate healthy messages to each population segmentation

\section{Critical Steps Within the Protocol:}

Critical steps within the protocol include the proper selection of baselines against which to assess the significance of the provided stimuli, as well as artifact rejection. Here, we used the IAPS database, which provide a large collection of images whose emotional reactions were evaluated as baseline, and the ICA methodology, which removed the artifacts induced by ocular and muscle movements from the EEG traces.

\section{Significance of the Obtained Data:}

The absence of a statistically significant effect of the gender factor in any index suggests that there is no difference between young males and females in the reaction to antismoking stimuli. This seems to be in line with the rather homogeneous spread of the smoking habit in the Italian population among the investigated age population ${ }^{74}$.

Concerning the AW index, the "awarded" campaign resulted promoted a significantly more approaching attitude than the "effective" and "ineffective" PSAs for both the image and spot stimuli. This can be explained by the symbolic communication style characterizing the "awarded" PSA. In addition, the statistically significant effect of the spot category, as well as the same tendency of the image category, are in line with the results presented in previous studies, where the right or left hemisphere activation-reflected by the different P300 amplitude variation-was modulated by exposure to neutral, promoting, and contrasting smoking messages stimuli ${ }^{75}$.

Concerning the effort index, the "effective" image was the least effortful. This can be explained by the very clear message conveyed by the image of the lady who underwent a tracheotomy, with the flanking sentence "don't tell people smoking is bad, show them." Conversely, the "awarded" image, depicting a cigarette symbolizing an artery full fatty deposits, obtained the highest effort levels, probably due to the lesser-known vascular consequences of smoking habits in the general population in comparison to the more famous pulmonary effects. Finally, the "ineffective" image, depicting a man with his son behind the sentence "Think, don't smoke," probably elicited quite high effort values due to the apparently poor relationship between the text and the picture, possibly inducing observers to try to understand the connection between these two elements. In contrast to the "effective" image, the video belonging to the same campaign reported the highest effort values, probably due to the complexity of the narrated story, in which a young man tells about the heart attack he got when he was just 45 years old, as well as all the consequences of that event, starting from the scar on his chest to the limitations in his everyday life. This result is in accordance with a recent study providing evidence that the presence of a narrative structure in video commercials results in higher theta power in the left frontal ${ }^{76}$.

Finally, the "effective" campaign showed the highest El values, possibly due to the empathy induced by the sick testimonials of the campaign and because of the explicitness of the message. This result could be in accordance with evidence obtained through a questionnaire in the same age group as our sample (16-19 years old). The questionnaire compared the perception of a fear-inducing antismoking image with the perception of a more positive image, and it showed that the participants preferred the fear-inducing one because it was more "appealing" than the first one $^{77}$. Furthermore, the higher El values estimated for the "effective" campaign, in agreement with a previous pilot study on antismoking TV commercials $^{11}$, is supported by the evidence that emotional anti-smoking advertisements have been associated with increased effectiveness, evaluated in terms of number of quit attempts in the adult population, in comparison to other kinds of advertising ${ }^{78}$.

A young population sample was selected because data concerning the epidemiology of tobacco consumption initiation in Italy mostly involves people from 15-17 years old ${ }^{74}$. Results obtained in the present study, although conducted on a limited sample of high school students, nevertheless support the usefulness of such neurometric indices to evaluate antismoking PSA evaluation. Further studies on a larger sample of students in the framework of the SmokeFreeBrain research program could return more decisive information on the generation of "effective" PSAs for young people. The presence of a fit between the target population and the advertised message has been shown to increase the impact of the message on implicit attitudes and correlated actions and to induce a more positive evaluation of the promoted message ${ }^{79}$. Finally, in this framework, one of the major factors of interest for the design of an effective campaign is the choice of the most appropriate testimonial ${ }^{80}$. Evidently, this investigation needs to be extended to an older sample, since the physiological, cognitive, and autonomic reaction to advertising and, in particular, antismoking PSAs could be different in different age populations ${ }^{27,81,82}$. Finally, the results of the present and future research will potentially reduce the waste of public money related to the generation and broadcasting of "ineffective" PSAs for different target populations.

\section{Disclosures}

The authors have nothing to disclose.

\section{Acknowledgements}

The present work was supported in part by the European Commission by Horizon2020 project HCO-06-2015, "SmokeFreeBrain," GA n. 681120.

\section{References}

1. Bargh, J.A., Chartrand, T.L. The unbearable automaticity of being. Am Psychol. 54 (7), $462-479$ (1999).

2. Damasio, A.R., Everitt, B.J., Bishop, D. The Somatic Marker Hypothesis and the Possible Functions of the Prefrontal Cortex [and Discussion]. Philos Trans R Soc Lond B Biol Sci. 351 (1346), 1413-1420 (1996). 
3. Davidson, R.J., Abercrombie, H., Nitschke, J.B., Putnam, K. Regional brain function, emotion and disorders of emotion. Curr Opin Neurobiol. 9 (2), 228-234 (1999).

4. Panksepp, J., Nocjar, C., Burgdorf, J., Panksepp, J.B., Huber, R. The role of emotional systems in addiction: a neuroethological perspective. Nebr Symp Motiv. 50, 85-126 (2004).

5. Ariely, D., Berns, G.S. Neuromarketing: the hope and hype of neuroimaging in business. Nat Rev Neurosci. 11 (4), $284-292$ (2010).

6. Baldo, D. et al. Brain Waves Predict Success of New Fashion Products: A Practical Application for the Footwear Retailing Industry. $J$ Creating Value. 1 (1), 61-71 (2015).

7. Plassmann, H., Ramsøy, T.Z., Milosavljevic, M. Branding the brain: A critical review and outlook. J Consum Psychol. 22, 18-36 (2012).

8. Lee, N., Broderick, A.J., Chamberlain, L. What is 'neuromarketing'? A discussion and agenda for future research. Int J Psychophysiol. 63 (2), 199-204 (2007).

9. Breiter, H.C. et al. Redefining neuromarketing as an integrated science of influence. Front Hum Neurosci. 8 (1073), 1-7 (2015).

10. Vecchiato, G. et al. Neurophysiological Tools to Investigate Consumer's Gender Differences during the Observation of TV Commercials. Comput Math Methods Med. 2014 (912981), 1-12 (2014).

11. Cartocci, G. et al. A pilot study on the neurometric evaluation of "effective" and "ineffective" antismoking public service announcements. 2016 38th Annual International Conference of the IEEE Engineering in Medicine and Biology Society (EMBC.). 4597-4600 (2016).

12. Han, S., Shavitt, S. Persuasion and Culture: Advertising Appeals in Individualistic and Collectivistic Societies. J Exp Soc Psychol. 30, 326-350 (1994).

13. Vecchiato, G. et al. On the Use of EEG or MEG Brain Imaging Tools in Neuromarketing Research. Comput Intell Neurosci. 2011 (643489) (2011).

14. Vecchiato, G. et al. Changes in Brain Activity During the Observation of TV Commercials by Using EEG, GSR and HR Measurements. Brain Topogr. 23 (2), 165-179 (2009).

15. Vecchiato, G. et al. Spectral EEG frontal asymmetries correlate with the experienced pleasantness of TV commercial advertisements. Med Biol Eng Comput. 49 (5), 579-583 (2011).

16. Cherubino, P. et al. Neuroelectrical Indexes for the Study of the Efficacy of TV Advertising Stimuli. Selected Issues in Experimental Economics, Springer Proceedings in Business and Economics. 355-371 (2016).

17. Paulus, M.P., Frank, L.R. Ventromedial prefrontal cortex activation is critical for preference judgments. Neuroreport. 14 (10), $1311-1315$ (2003).

18. Reimann, M., Schilke, O., Weber, B., Neuhaus, C., Zaichkowsky, J. Functional magnetic resonance imaging in consumer research: A review and application. Psychol Mark. 28 (6), 608-637 (2011).

19. Cherubino, P. et al. Marketing Meets Neuroscience: Useful Insights for Gender Subgroups During the Observation of TV Ads. Applying Neuroscience to Business Practice, IGI Global. 1-28 (2016).

20. Knutson, B., Rick, S., Wimmer, G.E., Prelec, D., Loewenstein, G. Neural predictors of purchases. Neuron. 53 (1), 147-156 (2007).

21. Coan, J.A., Allen, J.J. Frontal EEG asymmetry as a moderator and mediator of emotion. Biol Psychol. 67 (1-2), 7-49 (2004).

22. Davidson, R.J., Irwin, W. The functional neuroanatomy of emotion and affective style. Trends Cogn Sci. 3 (1), 1-21 (1999).

23. Davidson, R.J., Ekman, P., Saron, C.D., Senulis, J.A., Friesen, W.V. Approach-withdrawal and cerebral asymmetry: Emotional expression and brain physiology: I. J Pers Soc Psychol. 58, 330-341 (1990).

24. Davidson, R.J., Hugdahl, K. Brain Asymmetry. MIT Press. (1996).

25. Davidson, R.J. Affective style and affective disorders: perspectives from affective neuroscience. Cognition and Emotion. 12, 307-330 (1998).

26. Vecchiato, G., Kong, W., Maglione, A.G., Wei, D. Understanding the Impact of TV Commercials: Electrical Neuroimaging. IEEE Pulse. 3, 42-47 (2012).

27. Cartocci, G. et al. Gender and Age Related Effects While Watching TV Advertisements: An EEG Study. Comput Intell Neurosci. 2016. (2016).

28. Klimesch, W. EEG alpha and theta oscillations reflect cognitive and memory performance: a review and analysis. Brain Res Brain Res Rev. 29, 169-195 (1999).

29. Cartocci, G. et al. The "NeuroDante project": neurometric measurements of participant's reaction to literary auditory stimuli from Dante's "Divina Commedia". Symbiotic conference proceedings, Springer. In press, (2016).

30. Aricò, P. et al. Towards a multimodal bioelectrical framework for the online mental workload evaluation. 2014 36th Annual International Conference of the IEEE Engineering in Medicine and Biology Society. 3001-3004 (2014).

31. Wisniewski, M.G. et al. Frontal midline $\theta$ power as an index of listening effort. Neuroreport. 26, $94-99$ (2015).

32. Cartocci, G. et al. Mental workload estimations in unilateral deafened children. 2015 37th Annual International Conference of the IEEE Engineering in Medicine and Biology Society (EMBC). 1654-1657 (2015).

33. Gevins, A., Smith, M. E. Neurophysiological measures of cognitive workload during human-computer interaction. Theor Issues Ergon Sci. 4, 113-131 (2003).

34. Kato, J., Ide, H., Kabashima, I., Kadota, H., Takano, K., Kansaku, K. Neural Correlates of Attitude Change Following Positive and Negative Advertisements. Front Behav Neurosci. 3 (6), 1-13 (2009).

35. Berridge, K., Winkielman, P. What is an unconscious emotion? (The case for unconscious 'liking'). Cognition and Emotion. 17, 181-211 (2003).

36. Mauss, I.B., Robinson, M. D. Measures of emotion: A review. Cognition and Emotion. 23, 209-237 (2009).

37. Astolfi, L. et al. Neural basis for brain responses to TV commercials: a high-resolution EEG study. IEEE Trans Neural Syst Rehabil Eng. 16, $522-531$ (2008).

38. Vecchiato, G. et al. Neuroelectrical brain imaging tools for the study of the efficacy of TV advertising stimuli and their application to neuromarketing. Springer. Berlin, Germany. (2013).

39. Vecchiato, G. et al. Marketing and Neuroscience: How Electroencephalographic Tools Could Help to Design and Analyze Commercial Advertising Campaign. Micro \& Macro Marketing 2. II Mulino. (2014).

40. Hornik, R. Public Health Communication: Evidence for Behavior Change. Routledge. (2002).

41. Atusingwize, E., Lewis, S., Langley, T. Economic evaluations of tobacco control mass media campaigns: a systematic review. Tob Control. 24, 320-327 (2015).

42. Holtgrave, D.R., Wunderink, K.A., Vallone, D.M., Healton, C.G. Cost-Utility Analysis of the National truth® Campaign to Prevent Youth Smoking. Am J Prev Med. 36, 385-388 (2009).

43. Hurley, S.F., Matthews, J.P. Cost-effectiveness of the Australian National Tobacco Campaign. Tob Control. 17, 379-384 (2008).

44. Xu, X. et al. A Cost-Effectiveness Analysis of the First Federally Funded Antismoking Campaign. Am J Prev Med. 48, 318-325, (2015). 
45. Cartocci, G. et al. Frontiers | Against smoking public service announcements, a neurometric evaluation of effectiveness. <http:// www.frontiersin.org/10.3389/conf.fnhum.2016.220.00096/event_abstract> (2016).

46. De Pascalis, V., Cozzuto, G., Caprara, G.V., Alessandri, G. Relations among EEG-alpha asymmetry, BIS/BAS, and dispositional optimism. Biol Psychol. 94 (1), 198-209 (2013).

47. Jasper, H.H. The 10/20 international electrode system. EEG and Clinical Neurophysiology. 10, 371-375 (1958).

48. Boucsein, W. et al. Publication recommendations for electrodermal measurements. Psychophysiology. 49, 1017-1034 (2012).

49. Lang, P.J., Bradley, M.M., Cuthbert, B.N. International affective picture system (IAPS): Affective ratings of pictures and instruction manual. Technical report $A-8$. <https://www.researchgate.net/ publication/256309451_International_Affective_Picture_System_IAPS_Affective_Ratings_of_Pictures_and_Instruction_Manual_Rep_No_A-8> (2008).

50. Delorme, A., Makeig, S. EEGLAB: an open source toolbox for analysis of single-trial EEG dynamics including independent component analysis. J Neurosci Methods. 134 (1), 9-21 (2004).

51. Benedek, M., Kaernbach, C.A continuous measure of phasic electrodermal activity. J Neurosci Methods. 190, 80-91 (2010).

52. Lee, T.W., Girolami, M., Sejnowski, T.J. Independent component analysis using an extended infomax algorithm for mixed subgaussian and supergaussian sources. Neural comput. 11 (2), 417-441 (1999).

53. Davidson, R.J. What does the prefrontal cortex 'do' in affect: perspectives on frontal EEG asymmetry research. Biol Psychol. 67 (1-2), 219-234 (2004).

54. Borghini, G. et al. Avionic technology testing by using a cognitive neurometric index: A study with professional helicopter pilots. 201537 th Annual International Conference of the IEEE Engineering in Medicine and Biology Society (EMBC). 6182-6185 (2015).

55. Maglione, A. G. et al. Alpha EEG Frontal Asymmetries during Audiovisual Perception in Cochlear Implant Users. A Study with Bilateral and Unilateral Young Users. Methods Inf Med. 54, 500-504 (2015).

56. Schmidt, B., Hanslmayr, S. Resting frontal EEG alpha-asymmetry predicts the evaluation of affective musical stimuli. Neurosci Lett. 460 (3), 237-240, (2009)

57. Harmon-Jones, E., Gable, P.A., Peterson, C.K. The role of asymmetric frontal cortical activity in emotion-related phenomena: a review and update. Biol Psychol. 84 (3), 451-462 (2010).

58. Di Flumeri, G. et al. EEG Frontal Asymmetry Related to Pleasantness of Olfactory Stimuli in Young Subjects. Selected Issues in Experimental Economics. 373-381. Springer International Publishing. (2016).

59. Sutton, S.K., Davidson, R.J. Prefrontal brain electrical asymmetry predicts the evaluation of affective stimuli. Neuropsychologia. 38 (13), 1723-1733 (2000).

60. Vecchiato, G. et al. How to Measure Cerebral Correlates of Emotions in Marketing Relevant Tasks. Cog Comp. 6 (4), $856-871$ (2014).

61. Pan, J. Tompkins, W.J. A Real-Time QRS Detection Algorithm. IEEE Trans Biomed Eng. BME-32, 230-236 (1985).

62. Fowles, D. C. et al. Committee report. Publication recommendations for electrodermal measurements. Psychophysiology. 18, 232-239 (1981).

63. Russell, J.A., Barrett, L.F. Core affect, prototypical emotional episodes, and other things called emotion: Dissecting the elephant. J Pers Soc Psychol. 76, 805-819 (1999).

64. Posner, J., Russell, J.A., Peterson, B.S. The circumplex model of affect: An integrative approach to affective neuroscience, cognitive development, and psychopathology. Dev Psychopathol. 17, 715-734 (2005).

65. Coffman, J. Public communication campaign evaluation. Communications Consortium Media Center. Washington, DC. (2002).

66. Varcoe, J. Assessing the effectiveness of social marketing. Public Sector Research Conference. ESOMAR. (2004).

67. Centers for Disease Control and Prevention. CDC: Tips from Former Smokers - Roosevelt's Ad. <https://www.youtube.com/watch? $\mathrm{v}=$ Odml35elnCQ> (2017).

68. Duke, J.C. et al. Impact of a U.S. antismoking national media campaign on beliefs, cognitions and quit intentions. Health Educ Res. 30 (3), 466-483 (2015).

69. Think Don't Smoke. <https://www.youtube.com/watch?v=3B133Es-CKA> (2017).

70. Farrelly, M.C., Healton, C.G., Davis, K.C., Messeri, P., Hersey, J.C., Haviland, M.L. Getting to the Truth: Evaluating National Tobacco Countermarketing Campaigns. Am J Public Health. 92 (6), 901-907 (2002).

71. Biener, L. Anti-tobacco advertisements by Massachusetts and Philip Morris: what teenagers think. Tob Control. 11 (suppl 2), ii43-ii46 (2002).

72. British Heart Foundation. British Heart Foundation - Fatty Cigarette TV advert 2004. <https://www. youtube.com/watch?v=cDAN7Oi62e0> (2017).

73. Zar, J. Biostatistical Analysis. Prentice Hall. New York. (2000).

74. Il fumo in Italia - ISS-DOXA 2015. <http://www.iss.it/fumo/index.php?lang=1\&id=350\&tipo=18> (2016).

75. Jang, K.W., Lee, J.S., Yang, B.H., Lee, J.H. Changes of Brain Potentials in Response to Smoking-Induced Stimuli in Smokers. CyberPsychol Behav. 10, 460-463 (2007).

76. Wang, R. W.Y., Chang, Y.C., Chuang, S.W. EEG Spectral Dynamics of Video Commercials: Impact of the Narrative on the Branding Product Preference. Sci Rep. 6 (36487), 1-11 (2016).

77. Montazeri, A., McEwen, J. Effective communication: perception of two anti-smoking advertisements. Patient Educ Couns. 30, 29-35 (1997).

78. Farrelly, M.C. et al. Promotion of Smoking Cessation with Emotional and/or Graphic Antismoking Advertising. Am J Prev Med. 43, 475-482 (2012).

79. Mannetti, L., Brizi, A., Giacomantonio, M., Higgins, E. T. Framing political messages to fit the audience's regulatory orientation: How to improve the efficacy of the same message content. PloS One. 8 (10), e77040 (2013).

80. Mannetti, L., Brizi, A., Belanger, J., Bufalari, I. All we need is the candidate's face: The irrelevance of information about political coalition affiliation and campaign promises. Cogent Psychol. 3 (1268365), 1-12 (2016).

81. Bala, M.M., Strzeszynski, L., Topor-Madry, R., Cahill, K. Mass media interventions for smoking cessation in adults. Cochrane Database Syst Rev. 1 (CD004704) (2013).

82. Brinn, M.P., Carson, K.V., Esterman, A.J., Chang, A.B., Smith, B.J. Mass media interventions for preventing smoking in young people. Cochrane Database Syst Rev. 11 (CD001006) (2010). 